\title{
Memory Part 3: The Role of the Fornix and Clinical Cases
}

\author{
F.D. Raslau, J.C. Augustinack, A.P. Klein, (D).L. Ulmer, V.P. Mathews, and (DL.P. Mark
}

T he fornix is the major white matter outflow tract from the hippocampus, and pathology involving the fornix would be expected to affect memory. Pathologic processes that affect the hippocampal formation and parahippocampal gyrus, including the entorhinal cortex, correlate with specific types of memory deficits, ${ }^{1-10}$ but the clinical consequences of lesions of the fornix have not been emphasized as often. A familiarity with the anatomy and imaging of the fornix helps to anticipate the clinical impact of pathology and treatment that involves this strategic structure.

The fornix, named for its archlike configuration (Figs 1 and 2), is formed from the fimbria, which is the fringelike medial continuation of the alveus that sits on the superior surface of the hippocampus just below the ependymal lining on the floor of the temporal horn of the lateral ventricles (Fig 3). The posterior portion of the fornix, the crus, also forms the posterior medial margin of the lateral ventricles (Fig 4). The crura are joined across the midline by the psalterium (lyre or Harp of David), also called the hippocampal commissure or commissure of the fornix. ${ }^{11}$ The body of the fornix is situated at the dome of the fornical archlike configuration and is connected to the inner surface of the corpus callosum via the septum pellucidum (Fig $5 A$ ). This portion of the fornix is situated at the upper margin of the velum interpositum, which is traversed by the internal cerebral veins, and is above the third ventricle but below the body of the lateral ventricles. The foramen of Monro forms the anterior border of the velum interpositum, which is open at its posterior aspect at the position of the pineal gland (Fig 5B).

The white matter fibers of the columns of the fornix have a more superoinferior orientation, forming the anterior portion of the fornical arch, and are also joined to the posterior margin of the genu of the corpus callosum via the septum pellucidum (Fig 6). At

From the Department of Radiology (F.D.R.), University of Kentucky, Lexington, Kentucky; Department of Radiology (J.C.A.), Harvard Medical School, Boston, Massachusetts; Athinoula A. Martinos Center for Biomedical Imaging (J.C.A.), Massachusetts General Hospital and Harvard Medical School, Boston, Massachusetts; and Department of Radiology (A.P.K., J.L.U., V.P.M., L.P.M.), Medical College of Wisconsin, Milwaukee, Wisconsin.

Please address correspondence to Leighton P. Mark, MD, Froedtert Hospital, Department of Radiology, Neuroradiology Section, 9200 West Wisconsin Ave, Milwaukee, WI 53226; e-mail: Imark@mcw.edu

http://dx.doi.org/10.3174/ajnr.A4371

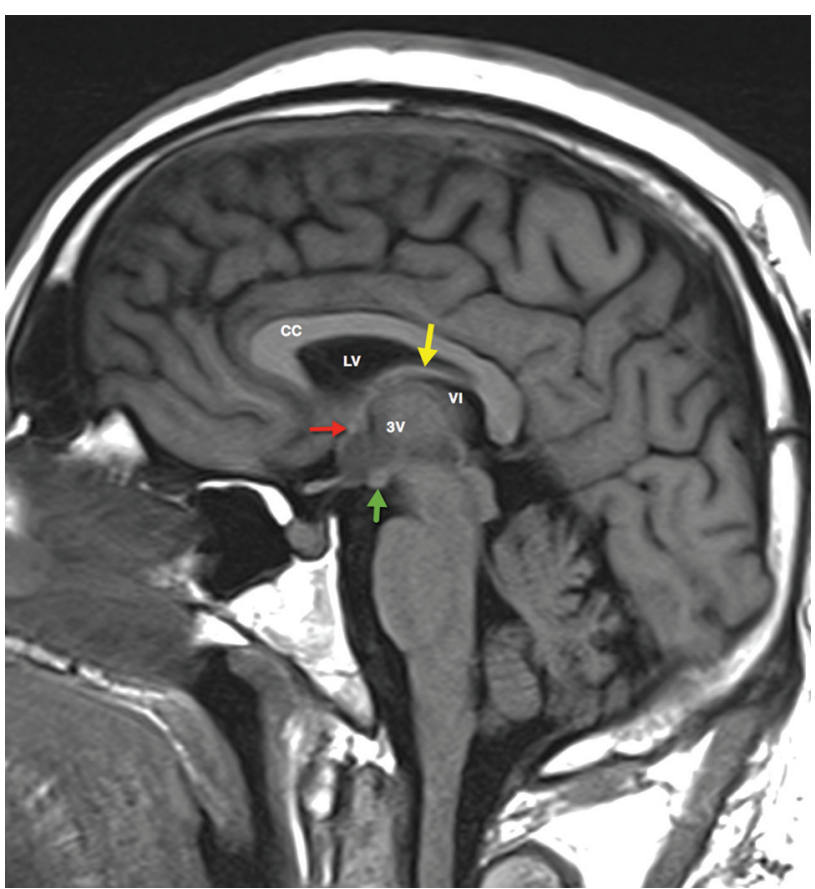

FIG 1. Sagittal T1-weighted image showing the archlike configuration of the fornix (yellow arrow) at the upper margin of the velum interpositum (VI), which is situated between the lateral (LV) and third (3V) ventricles. The fornix is attached to the septum pellucidum, which in turn is attached to the inner curving surface of the corpus callosum (CC). The fornix bifurcates at the level of the anterior commissure (red arrow), with the postcommissural fibers projecting to the mammillary bodies (green arrow).

the level of the anterior commissure, the columns of the fornix split, with most of the fibers coursing posterior to the anterior commissure to form the postcommissural fibers that project to the mammillary bodies, while the minority of precommissural fibers run anterior to the anterior commissure to project to the medial prefrontal cortex, nucleus accumbens, and septal nuclei, which are located deep to the medial aspect of the frontal lobes inferior to the rostrum of the corpus callosum. The fornix also sends smaller fibers to the anterior thalamus.

Clinical and experimental data suggest a functional distribution of the fornical fibers. ${ }^{12-16}$ The left fornix primarily carries verbal memory information, while the right carries visuospatial 


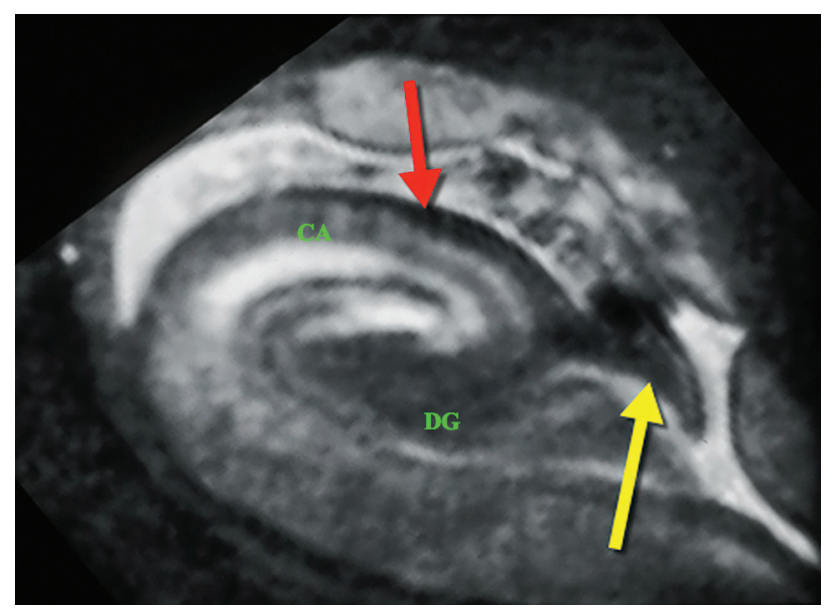

FIG 2. High-resolution coronal T2-weighted image of the right hippocampal formation showing the dark signal of the white matter fibers of the alveus (red arrow) positioned on top of Ammon's horn (CA) of the hippocampus and below the CSF of the temporal horn of the lateral ventricle, which also contains the choroid plexus (not labeled). The dentate gyrus (DG) is shown. The alveus thickens at its medial margin to form the fimbria (yellow arrow).

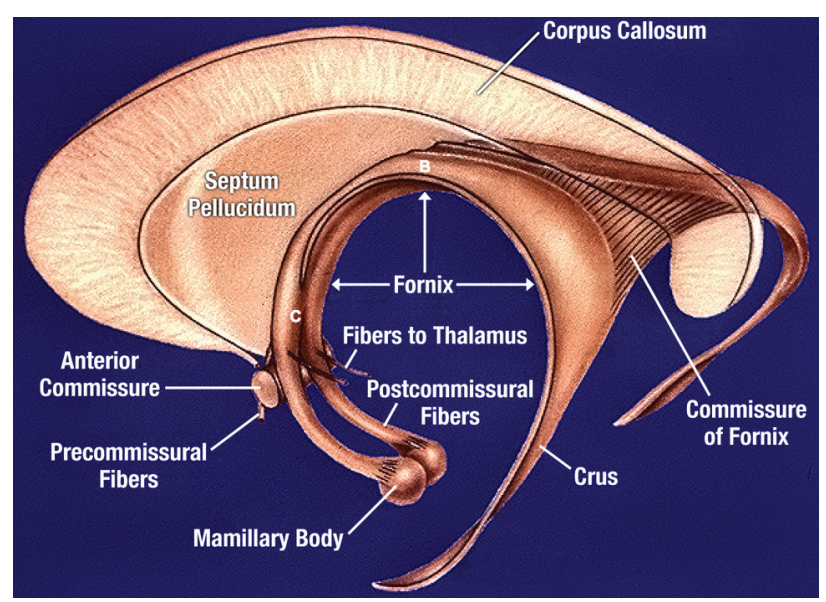

FIG 3. Schematic overview of the fornix from the side. The fimbria forms the crus of the fornix, which is joined to the opposite fornix across the midline by the commissure of the fornix (hippocampal commissure, psalterium). The body of the fornix (B) at the top of the fornical arch is connected to the inferior surface of the body of the corpus callosum by the septum pellucidum. The column of the fornix (C) has a more superoinferior orientation and is connected to the posterior margin of the genu of the corpus callosum by the septum pellucidum. The columns of the fornix are split at the level of the anterior commissure with most the fibers (postcommissural fibers) projecting to the mammillary bodies. Published with permission from the estate of David L. Daniels.

memory information (Fig 7). In addition, the medial fornix carries fibers from the caudal hippocampus, which processes exteroceptive signals and integrates object recognition within a spatial context (ie, scene learning). The lateral aspect of the fornix is presumed to carry projections from the more rostral hippocampus, which processes interoceptive signals for emotional and motivational learning and memory (Fig 8).

Knowledge of this anatomy helps to anticipate clinical deficits and surgical risks. Figure 9 shows the imaging of a 23-year-old woman who presented with progressive headaches over a 2 -month period. An unusual lesion was noted within the anterior
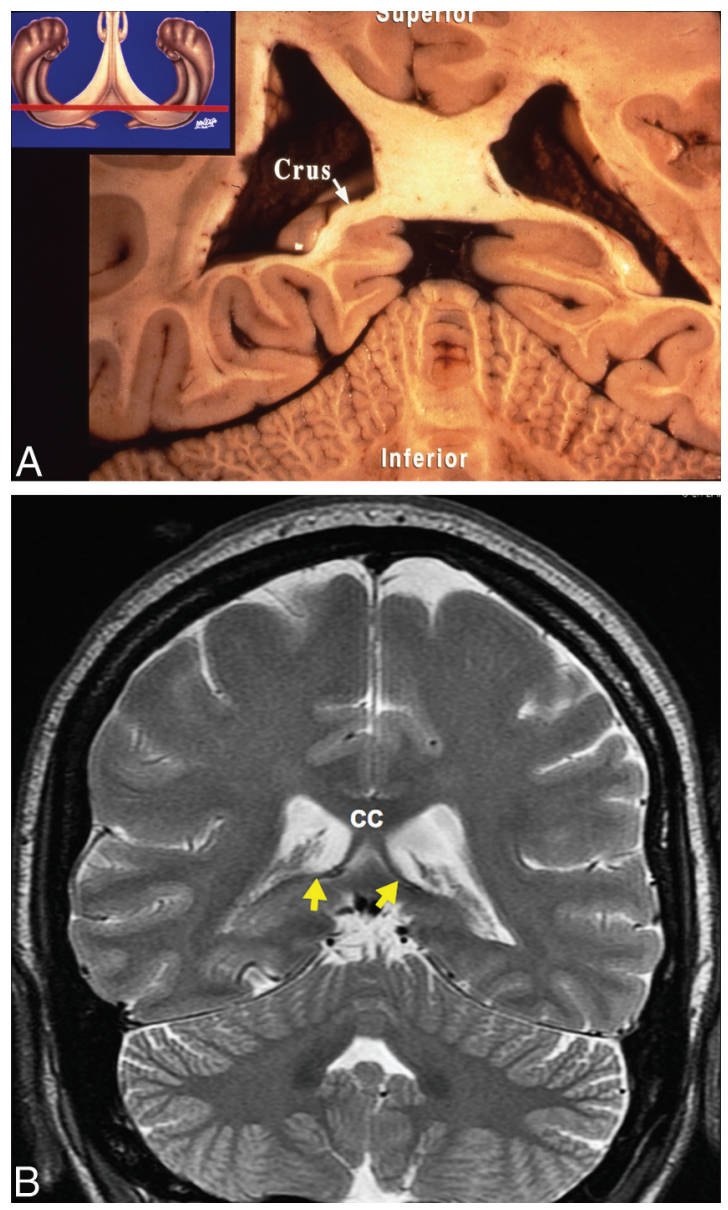

FIG 4. $A$, Coronal anatomic specimen with a schematic reference image. The white matter fibers of the crus of the fornix can be seen forming the inferior medial margin of the posterior aspect of the body of the lateral ventricles at the level of the splenium of the corpus callosum. B, Coronal T2-weighted image shows the dark signal of the crura of the fornices (yellow arrows) forming the inferior medial margin of the posterior lateral ventricles at the level of the posterior aspect of the body of the corpus callosum (CC).

right lateral ventricle, causing some obstruction of the foramen of Monro. More important, the mass impinged on and displaced the columns of the fornices, right more than left. The neurosurgeons were advised about the close proximity of the fornices to the medial margin of the tumor and the risk of memory impairment. The patient underwent an endoscopic subtotal resection, in which the lesion was discovered to be a glioneuronal neoplasm, which was tightly adherent to the fornical columns. On postoperative day 1 , the patient experienced new memory deficits with no recall of the surgery or reason for the surgery. Neuropsychological testing months later showed moderately impaired delayed recall of both verbal and visuospatial materials.

Figure $10 \mathrm{~A}$ shows an axial gradient-echo image of a 60 -yearold patient who presented with headaches and memory problems. A large collection of blood was noted in the cistern of the velum interpositum, with superior lateral displacement and compression of the body of the fornices. Cerebral angiography did not reveal any cause for the hemorrhage, and the patient was then monitored with serial MR imaging. Figure $10 B$ shows atrophy of the mammillary bodies, left greater than right, 5 months later. The hematoma had significantly diminished in size with time, but 

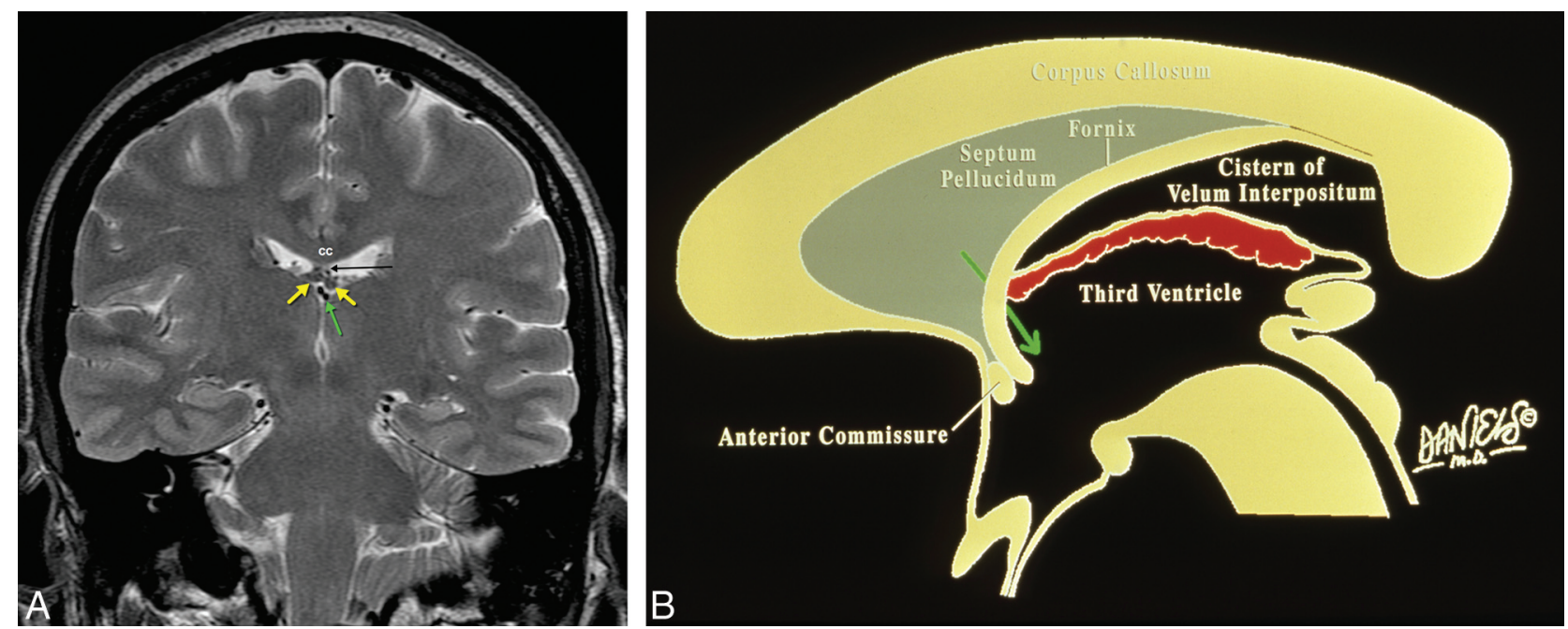

FIG 5. A, Coronal T2-weighted image shows the body of the fornices (yellow arrows) attached to the inferior margin of the body of the corpus callosum (CC) via the septum pellucidum (black arrow). The body of the fornices are at the upper aspect of the velum interpositum, which is traversed by the internal cerebral veins (green arrow), situated above the third ventricle but inferomedial to the body of the lateral ventricles. $B$, Sagittal schematic shows the position of the fornix relative to the velum interpositum. The foramen of Monro (green arrow) forms the anterior border of the velum interpositum, while the posterior border opens between the splenium of the corpus callosum above and the pineal gland below into the quadrigeminal plate cistern. The choroid plexus (red) is indicated at the roof of the third ventricle below but not within the velum interpositum. Published with permission from the estate of David L. Daniels.
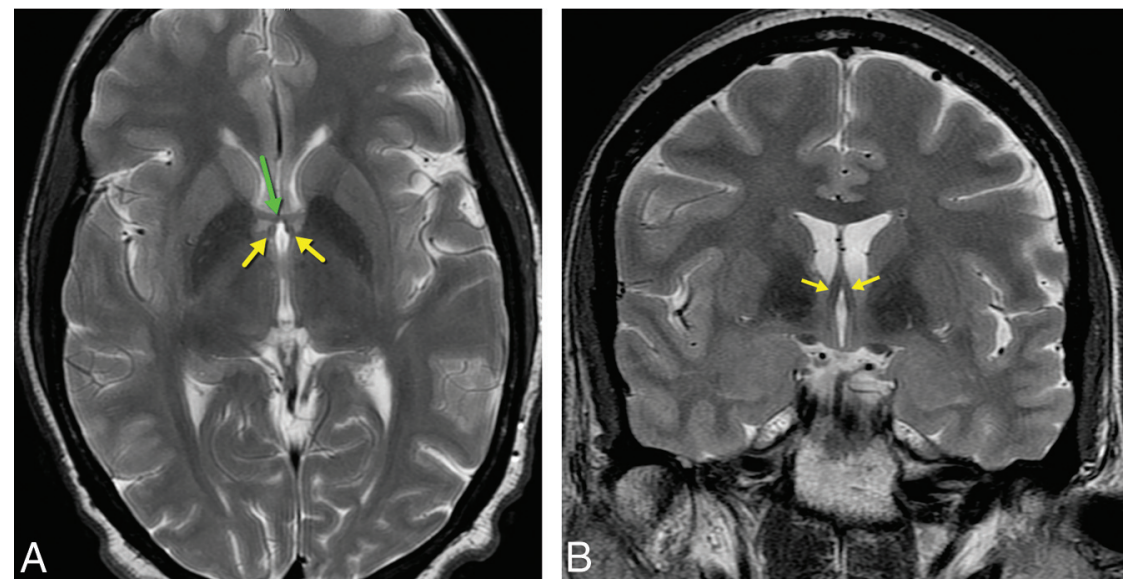

FIG 6. A, Axial T2-weighted image shows the postcommissural fibers of the fornix (yellow arrows) coursing posterior to the anterior commissure (green arrow). B, Coronal T2-weighted image shows the superoinferior orientation of the columns of the fornix (yellow arrows).

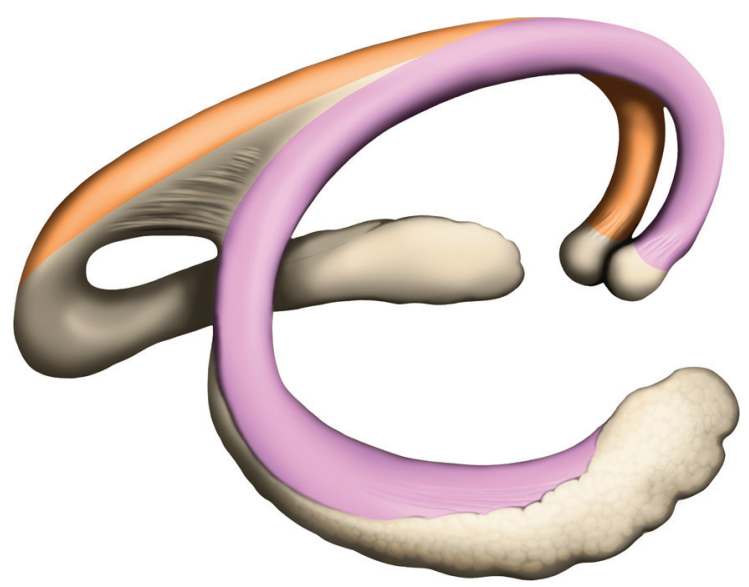

FIG 7. Drawing showing verbal memory information carried primarily by the left fornix (orange), while the visuospatial memory information is primarily transmitted by the right fornix (light purple).

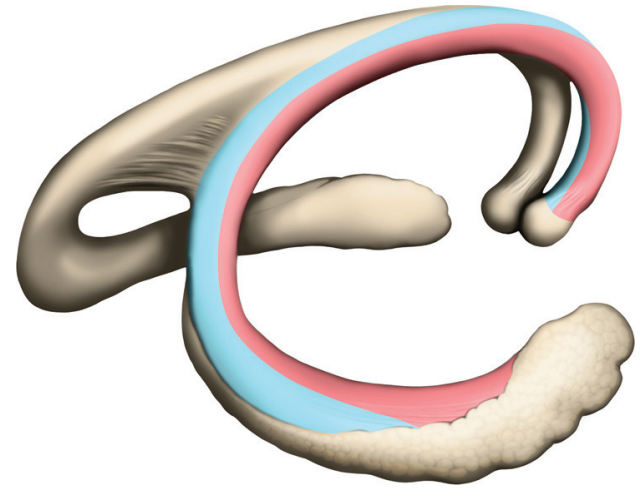

FIG 8. Drawing shows the medial aspect of the fornix (light blue) carrying fibers from the posterior hippocampus for processing of exteroceptive information for scene learning. The more lateral fibers (light red), meanwhile, carry fibers from the more rostral portions of the hippocampus for processing of interceptive signals for emotional and motivational memory and learning. 

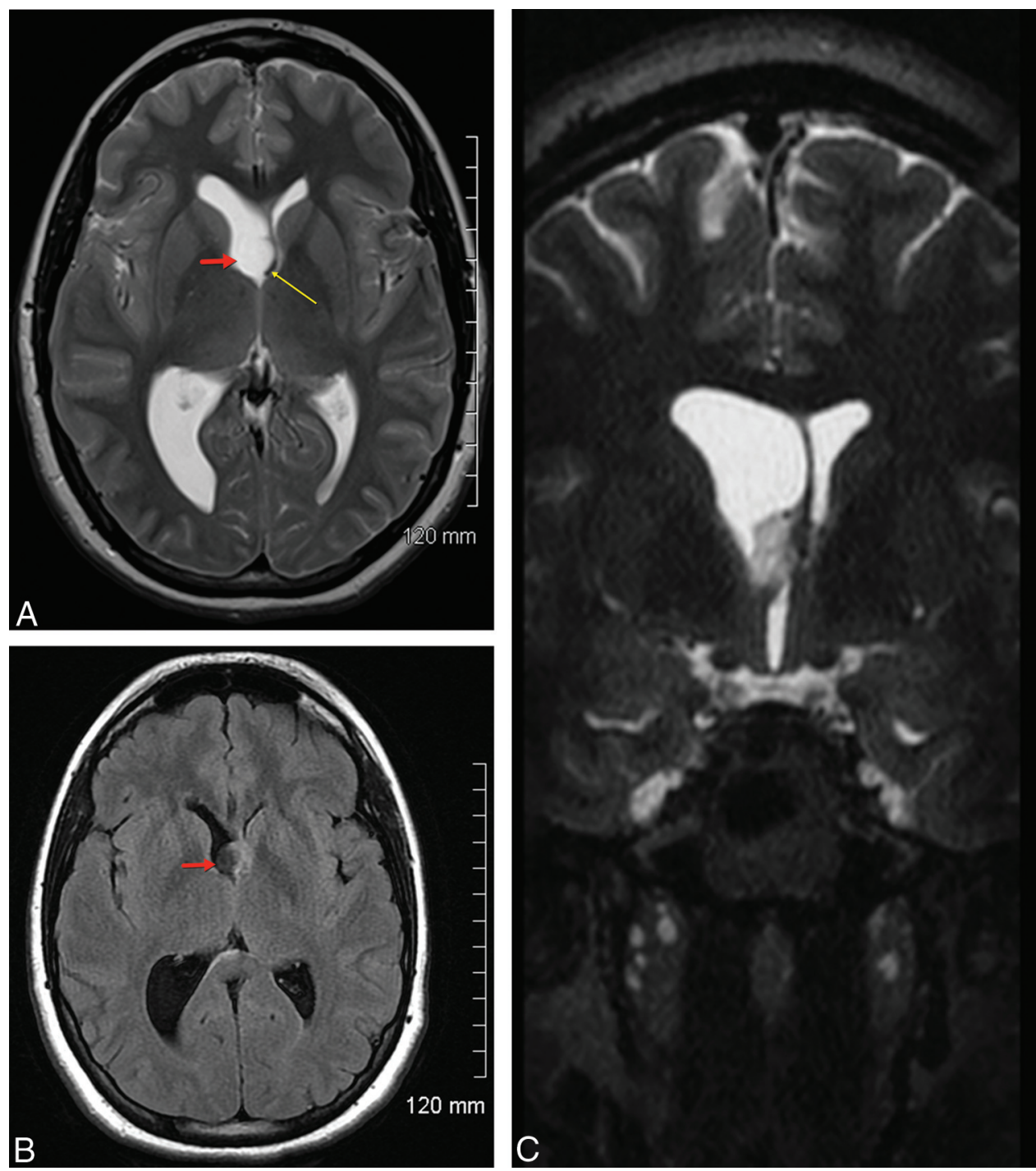

FIG 9. A, T2-weighted axial image shows a glioneuronal tumor (red arrow) within the right lateral ventricle near the foramen of Monro displacing and distorting the septum pellucidum and columns of the fornices (yellow arrow). B. The intraventricular glioneuronal tumor (red arrow) is better visualized on the axial FLAIR image. C, Postoperative coronal T2-weighted image shows the intraventricular neoplasm still distorting the columns of the fornix and septum pellucidum.
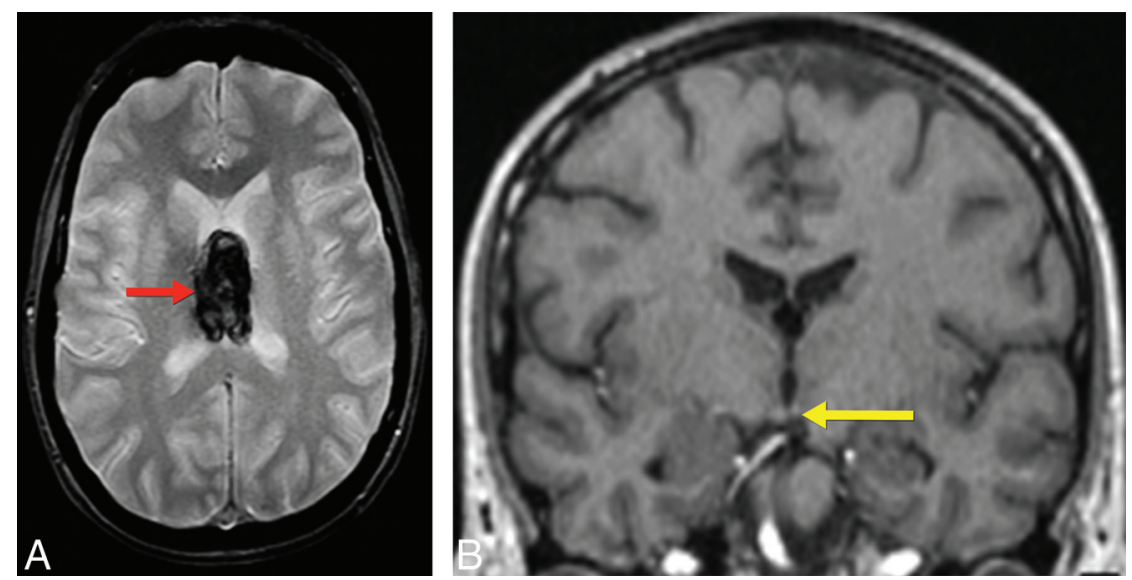

FIG 10. A, Gradient recalled-echo image shows hemorrhage (red arrow) in the cistern of the velum interpositum involving the bodies of the fornices. B, Coronal T1-weighted image 5 months later without treatment shows atrophy of the mammillary bodies, worse on the left (yellow arrow). 

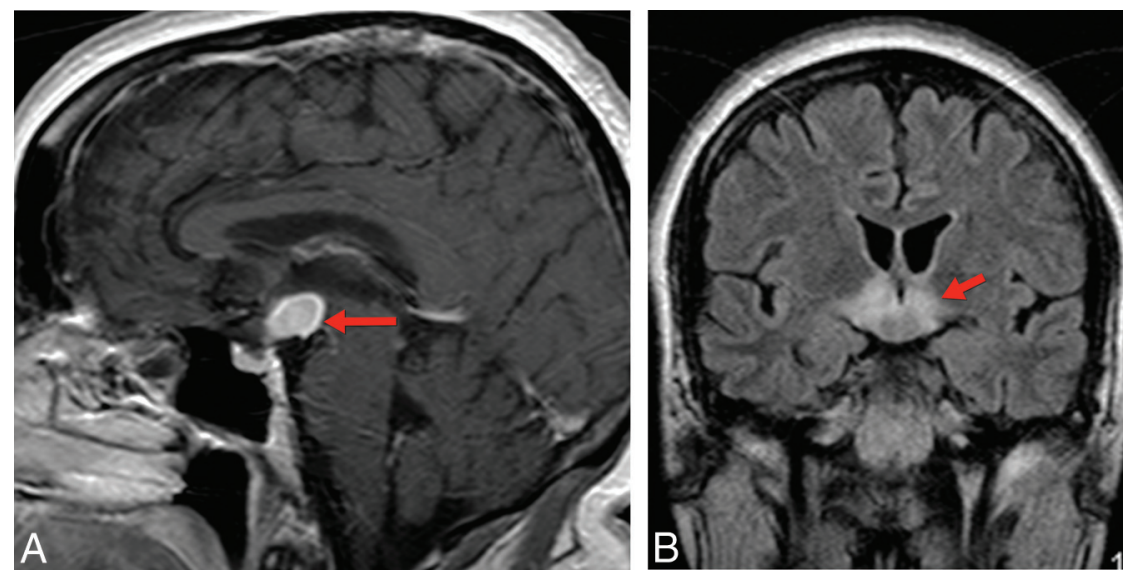

FIG 11. A, Sagittal T1 postcontrast image shows an enhancing mass involving the floor of the hypothalamus and mammillary body with extension into the third ventricle. $B$, Coronal FLAIR image shows extensive abnormal signal involving the hypothalamus on either side of the midline. Pathology (not shown) showed this to be Langerhans cell histiocytosis.

neuropsychological testing showed that the patient had residual memory impairment, verbal more than visuospatial. This is expected given the lateralization of the verbal memory fibers to the left fornix and its greater involvement by the hematoma as reflected in the smaller left mammillary body. Figure 11 shows the initial imaging of a 56-year-old woman who presented with new onset of memory loss. She would often repeat the same question during the appointment with her doctor, not remembering that her question had already been answered. The infiltrating enhancing lesion of her mammillary bodies, including much of the remainder of the hypothalamus demonstrated on the MR imaging examination, proved to be Langerhans cell histiocytosis.

The effort of investigations in the basic neurosciences will continue to expand and deepen our knowledge of the processes that constitute the experience that is memory, and our everyday clinical effort to understand the functional anatomy of the extended hippocampal network that subserves memory is useful to predict the dysfunctional implications of strategically located lesions and enhance the role of the neuroradiolgist in diagnosis and guiding treatment.

\section{ACKNOWLEDGMENTS}

In memory of David L. Daniels, MD, whose medical artwork continues to inspire us.

Disclosures: Vincent P. Mathews_UNRELATED: Payment for Lectures (including service on Speakers Bureaus): Eli Lilly, Comments: speakers bureau for Amyvid in 2013.

\section{REFERENCES}

1. Augustinack JC, Huber KE, Postelnicu GM, et al. Entorhinal verrucae geometry is coincident and correlates with Alzheimer's lesions: a combined neuropathology and high-resolution ex vivo MRI analysis. Acta Neuropathol 2012;123:85-96

2. Augustinack JC, Schneider A, Mandelkow EM, et al. Specific tau phosphorylation sites correlate with severity of neuronal cytopathology in Alzheimer's disease. Acta Neuropathol 2002;103:26-35
3. Augustinack JC, van der Kouwe AJ, Salat DH, et al. H.M.'s contributions to neuroscience: a review and autopsy studies. Hippocampus 2014;24:1267-86

4. Bannerman DM, Rawlins JN, McHugh SB, et al. Regional dissociations within the hippocampus: memory and anxiety. Neurosci Biobehav Rev 2004;28:273-83

5. Block RI, O'Leary DS, Ehrhardt JC, et al. Effects of frequent marijuana use on brain tissue volume and composition. Neuroreport 2000;11:491-96

6. Block RI, O'Leary DS, Hichwa RD, et al. Effects of frequent marijuana use on memory-related regional cerebral blood flow. Pharmacol Biochem Behav 2002;72:237-50

7. Dickerson BC, Feczko E, Augustinack JC, et al. Differential effects of aging and Alzheimer's disease on medial temporal lobe cortical thickness and surface area. Neurobiol Aging 2009;30:432-40

8. Moser MB, Moser EI. Functional differentiation in the hippocampus. Hippocampus 1998;8:608-19

9. Van Hoesen GW, Augustinack JC, Dierking J, et al. The parahippocampal gyrus in Alzheimer's disease: clinical and preclinical neuroanatomical correlates. Ann N Y Acad Sci 2000;911; 254-74

10. Van Hoesen, G, Augustinack J, Redman S, Ventromedial temporal lobe pathology in dementia, brain trauma, and schizophrenia. Ann N Y Acad Sci 1999;877: 575-94

11. Gloor P, Salanova V, Olivier A, et al., The human dorsal hippocampal commissure: an anatomically identifiable and functional pathway. Brain 1993;116(pt 5):1249-73

12. Cameron AS, Archibald YM. Verbal memory deficit after left fornix removal: a case report. Int J Neurosci 1981;12:201

13. Hodges JR, Carpenter K. Anterograde amnesia with fornix damage following removal of IIIrd ventricle colloid cyst. J Neurol Neurosurg Psychiatry 1991;54:633-38

14. McMackin D, Cockburn J, Anslow P, et al. Correlation of fornix damage with memory impairment in six cases of colloid cyst removal. Acta Neurochir (Wien) 1996;135:12-18

15. Saunders RC, Aggleton JP. Origin and topography of fibers contributing to the fornix in macaque monkeys. Hippocampus 2007;17: 396-411

16. Tucker DM, Roeltgen DP, Tully R, et al. Memory dysfunction following unilateral transection of the fornix: a hippocampal disconnection syndrome. Cortex 1988;24:465-472 